\title{
Practical Security Analysis of PUF-Based Two-Player Protocols
}

\author{
Ulrich Rührmair ${ }^{1}$ and Marten van Dijk ${ }^{2}$ \\ 1 Technische Universität München, 80333 München, Germany \\ ruehrmair@in.tum.de \\ 2 RSA Laboratories, Cambridge, MA, USA \\ marten.vandijk@rsa.com
}

\begin{abstract}
In recent years, PUF-based schemes have not only been suggested for the basic tasks of tamper sensitive key storage or the identification of hardware systems, but also for more complex protocols like oblivious transfer (OT) or bit commitment (BC), both of which possess broad and diverse applications. In this paper, we continue this line of research. We first present an attack on two recent OT- and BC-protocols which have been introduced at CRYPTO 2011 by Brzuska et al. [1|2]. The attack quadratically reduces the number of CRPs which malicious players must read out in order to cheat, and fully operates within the original communication model of [1]2]. In practice, this leads to insecure protocols when electrical PUFs with a medium challenge-length are used (e.g., 64 bits), or whenever optical PUFs are employed. These two PUF types are currently among the most popular designs. Secondly, we discuss countermeasures against the attack, and show that interactive hashing is suited to enhance the security of PUF-based OT and BC, albeit at the price of an increased round complexity.
\end{abstract}

Keywords: Physical Unclonable Functions (PUFs), Cryptographic Protocols, Oblivious Transfer, Bit Commitment, Security Analysis, Interactive Hashing.

\section{Introduction}

Today's electronic devices are mobile, cross-linked and pervasive, which makes them a well-accessible target for adversaries. The well-known protective cryptographic techniques all rest on the concept of a secret binary key: They presuppose that devices store a piece of digital information that is, and remains, unknown to an adversary. It turns out that this requirement is difficult to realize in practice. Physical attacks such as invasive, semi-invasive or side-channel attacks carried out by adversaries with one-time physical access to the devices, as well as software attacks like application programming interface (API) attacks, viruses or Trojan horses, can lead to key exposure and security breaks. As Ron Rivest emphasized in his keynote talk at CRYPTO 2011 [22], merely calling a bit string a "secret key" does not make it secret, but rather identifies it as an interesting target for the adversary.

Indeed, one main motivation for the development of Physical Unclonable Functions (PUFs) was their promise to better protect secret keys. A PUF is an (at least partly) disordered physical system $P$ that can be challenged with so-called external stimuli or

E. Prouff and P. Schaumont (Eds.): CHES 2012, LNCS 7428, pp. 251-267, 2012.

(C) International Association for Cryptologic Research 2012 
challenges $c$, upon which it reacts with corresponding responses $r$. Contrary to standard digital systems, these responses depend on the micro- or nanoscale structural disorder of the PUF. It is assumed that this disorder cannot be cloned or reproduced exactly, not even by the PUF's original manufacturer, and that it is unique to each PUF. Any PUF $P$ thus implements a unique and individual function $f_{P}$ that maps challenges $c$ to responses $r=f_{P}(c)$. The tuples $(c, r)$ are called the challenge-response pairs (CRPs) of the PUF.

Due to its complex internal structure, a PUF can avoid some of the shortcomings of classical digital keys. It is usually harder to read out, predict, or derive PUF-responses than to obtain digital keys that are stored in non-volatile memory. The PUF-responses are only generated when needed, which means that no secret keys are present permanently in the system in an easily accessible digital form. Finally, certain types of PUFs are naturally tamper sensitive: Their exact behavior depends on minuscule manufacturing irregularities, often in different layers of the IC, and removing or penetrating these layers will automatically change the PUF's read-out values. These facts have been exploited in the past for different PUF-based security protocols. Prominent examples include identification [21|9], key exchange [21], and various forms of (tamper sensitive) key storage and applications thereof, such as intellectual property protection or readproof memory [11|14|29].

In recent years, also the use of PUFs in more advanced cryptographic protocols together with formal security proofs has been investigated. In these protocols, usually PUFs with a large challenge set and with a freely accessible challenge-response interface are employed The PUF is used similar to a "physical random oracle", which is transferred between the parties, and which can be read-out exactly by the very party who currently holds physical possession of it. Its input-output behavior is assumed to be so complex that its response to a randomly chosen challenge cannot be predicted numerically and without direct physical measurement, not even by a person who had physical access to the PUF at earlier points in time. In 2010, Rührmair [23] showed that oblivious transfer (OT) can be realized between two parties by physically transferring a PUF in this setting. He observed that via the classical reductions of Kilian [13], this implies PUF-based bit commitment and PUF-based secure multi-party computations. In the same year, the first formal security proof for a PUF-protocol was provided by Rührmair, Busch and Katzenbeisser [24]. They presented definitions and a reductionist security proof for PUF-based identification protocols. At CRYPTO 2011 Brzuska et al. [1] adapted Canetti's universal composition (UC) framework [3] to include PUFs. They gave PUF-based protocols for oblivious transfer (OT), bit commitment (BC) and key exchange (KE) and proved them to be secure in their framework.

The investigation of advanced cryptographic settings for PUF makes sense even from the perspective of a pure practitioner: Firstly, it clarifies the potential of PUFs in theory, a necessary prerequisite before this potential can be unleashed in commercial applications without risking security failures. Secondly, BC and OT protocols are

${ }^{1}$ This type of PUF sometimes has been termed Physical Random Function [9] or Strong PUF $[11 \mid 26[25,24]$ in the literature. We emphasize that the Weak/Strong PUF terminology introduced by Guajardo et al. [11] is not to be understood in a judgemental or pejorative manner. 
extremely versatile cryptographic primitives, which allow the implementation of such diverse tasks as zero-knowledge identification, the enforcement of semi-honest behavior in cryptographic protocols, secure multi-party computation (including online auctions or electronic voting), or key exchange. If these tasks shall be realized securely in practice by PUFs, a theoretical investigation of the underlying primitives - in this case BC and $\mathrm{OT}-$ is required first.

In this paper, we continue this line of research, and revisit the use of PUFs in OTand BC-protocols. Particular emphasis is placed on the achievable practical security if well-established PUFs (like electrical PUFs with 64-bit challenge lengths or optical PUFs) are used in the protocols. We start by observing an attack on the OT- and BCprotocols of Brzuska et al. [12] which quadratically reduces the number of responses that a malicious player must read out in order to cheat. It works fully in the original communication model of Brzuska et al. and makes no additional assumptions. As we show, the attack makes the protocols insecure in practice if electrical PUFs with medium bitlengths around 64 bits are used, and generally if optical PUFs are employed. This has a special relevance since the use of optical PUFs for their protocols had been explicitly proposed by Brzuska et al. (see Section 8 of [2]). Secondly, we investigate countermeasures against our attack, and show that interactive hashing can be used to enhance the security of PUF-based OT and BC protocols.

Our work continues the recent trend of a formalization of PUFs, including protocol analyses, more detailed investigations of non-trivial communication settings, and formal security proofs. This trend will eventually lay the foundations for future PUF research, and seems indispensible for a healthy long-term development of the field. It also combines protocol design and practical security analyses in a novel manner.

Organization of this Paper. In Section 2 we present the protocols of Brzuska et al. in order to achieve a self-contained treatment. Section 3 gives our quadratic attack. Section 4 discusses its practical effect. Section 5 discusses countermeasures. We conclude the paper in Section 6 .

\section{The Protocols of Brzuska et al.}

Our aim in this paper is to present a quadratic attack on two recent PUF-protocols for OT and BC by Brzuska et al. [12] and to discuss its practical relevance. In order to achieve a self-contained treatment, we will now present these two protocols. To keep our exposition simple, we will not use the full UC-notation of [1], and will present the schemes mostly without error correction mechanisms, since the latter play no role in the context of our attack.

The protocols use two communication channels between the communication partners: A binary channel, over which all digital communication is handled. It is assumed that this channel is non-confidential, but authenticated. And secondly an insecure physical channel, over which the PUF is sent. It is assumed that adversaries can measure adaptively selected CRPs of the PUF while it is in transition over this channel. 


\subsection{Oblivious Transfer}

The OT protocol of [1] implements one-out-of-two string oblivious transfer. It is assumed that in each subsession the sender $P_{i}$ initially holds two (fresh) bitstrings $s_{0}, s_{1} \in$ $\{0,1\}^{\lambda}$, and that the receiver $P_{j}$ holds a (fresh) choice bit $b$.

Brzuska et al. generally assume in their treatment that after error correction and the application of fuzzy extractors, a PUF can be modeled as a function PUF : $\{0,1\}^{\lambda} \rightarrow$ $\{0,1\}^{r g(\lambda)}$. We use this model throughout this paper, too. In the subsequent protocol of Brzuska et al., it is furthermore assumed that $\operatorname{rg}(\lambda)=\lambda$, i.e., that the PUF implements a function PUF $:\{0,1\}^{\lambda} \rightarrow\{0,1\}^{\lambda}$ (compare [12]).

\section{Protocol 1: PUF-Based Oblivious Transfer ([1], Slightly Simplified De- SCRIPTION)}

External Parameters: The protocol has a number of external parameters, including the security parameter $\lambda$, the session identifier sid, a number $N$ that specifies how many subsessions are allowed, and a pre-specified PUF-family $\mathcal{P}$, from which all PUFs which are used in the protocol must be drawn.

Initialization Phase: Execute once with fixed session identifier sid:

1. The receiver holds a PUF which has been drawn from the family $\mathcal{P}$.

2. The receiver measures $l$ randomly chosen CRPs $c_{1}, r_{1}, \ldots, c_{l}, r_{l}$ from the PUF, and puts them in a list $\mathcal{L}:=\left(c_{1}, r_{1}, \ldots, c_{l}, r_{l}\right)$.

3. The receiver sends the PUF to the sender.

Subsession Phase: Repeat at most $N$ times with fresh subsession identifier ssid:

1. The sender's input are two strings $s_{0}, s_{1} \in\{0,1\}^{\lambda}$, and the receiver's input is a bit $b \in\{0,1\}$.

2. The receiver chooses a CRP $(c, r)$ from the list $\mathcal{L}$ at random.

3. The sender chooses two random bitstrings $x_{0}, x_{1} \in\{0,1\}^{\lambda}$ and sends $x_{0}, x_{1}$ to the receiver.

4. The receiver returns the value $v:=c \oplus x_{b}$ to the sender.

5. The sender measures the responses $r_{0}$ and $r_{1}$ of the PUF that correspond to the challenges $c_{0}:=v \oplus x_{0}$ and $c_{1}:=v \oplus x_{1}$.

6. The sender sets the values $S_{0}:=s_{0} \oplus r_{0}$ and $S_{1}:=s_{1} \oplus r_{1}$, and sends $S_{0}, S_{1}$ to the receiver.

7. The receiver recovers the string $s_{b}$ that depends on his choice bit $b$ as $s_{b}=S_{b} \oplus r$. He erases the pair $(c, r)$ from the list $\mathcal{L}$.

Comments. The protocol implicitly assumes that the sender and receiver can interrogate the PUF whenever they have access to it, i.e., that the PUF's challenge-response interface is publicly accessible and not protected. This implies that the employed PUF must possess a large number of CRPs. Using a PUF with just a few challenges does not make sense: The receiver could then create a full look-up table for all CRPs of such a PUF 
before sending it away in Step 3 of the Initialization Phase. This would subsequently allow him to recover both strings $s_{0}$ and $s_{1}$ in Step 6 of the protocol subsession, as he could obtain $r_{0}$ and $r_{1}$ from his look-up table. Similar observations hold for the upcoming protocol 2. Indeed, all protocols discussed in this paper require PUFs with a large number of challenges and publicly accessible challenge-response interfaces. These PUFs have sometimes been referred to as Physical Random Functions or also as Strong PUFs in the literature [11 26|25].

Furthermore, please note that no physical transfer of the PUF is envisaged during the subsessions of the protocol. According to the model of Brzuska et al., an adversary only has access to it during the initialization phase, but not between the subsessions. This protocol use has some similarities with a stand-alone usage of the PUF, in which exactly one PUF-transfer occurs between the parties.

\subsection{Bit Commitment}

The second protocol of [1] implements PUF-based Bit Commitment (BC) by a generic reduction to PUF-based OT. The BC-sender initially holds a bit $b$. When the OTProtocol is called as a subprotocol, the roles of the sender and receiver are reversed: The BC-sender acts as the OT-receiver, and the BC-receiver as the OT-sender. The details are as follows.

Protocol 2: PUF-BASEd Bit COMMITMENT VIA PUF-BASED OBLIVIOUS TRANSFER ([1], SLightly Simplified DesCription)

\section{Commit Phase:}

1. The BC-sender and the BC-receiver jointly run an OT-protocol (for example Protocol 1).

(a) In this OT-protocol, the BC-sender acts as OT-receiver and uses his bit $b$ as the choice bit of the OT-protocol.

(b) The BC-receiver acts as OT-sender. He chooses two strings $s_{0}, s_{1} \in\{0,1\}^{\lambda}$ at random, and uses them as his input $s_{0}, s_{1}$ to the OT-protocol.

2. When the OT-protocol is completed, The BC-sender has learned the string $v:=s_{b}$. This closes the commit phase.

\section{Reveal Phase:}

1. In order to reveal bit $b$, the BC-sender sends the string $(b, v)$ (with $v=s_{b}$ ) to the BC-receiver.

Comments. The security of the BC-protocol is inherited from the underlying OT-protocol. Once this protocol is broken, also the security of the BC-protocol is lost. This will be relevant in the upcoming sections. 


\section{A Quadratic Attack on Protocols 1 and 2}

We will now discuss a cheating strategy in Protocols 1 and 2 Compared to an attacker who exhaustively queries the PUF for all of its $m$ possible challenges, we describe an attack on Protocols 1 and 2 which reduces this number to $\sqrt{m}$. As we will argue later in Section 4, this has a particularly strong effect on the protocol's security if an optical PUF is used (as has been explicitly suggested by [2]), or if electrical PUFs with medium challenge lengths of 64 bits are used.

Our attack rests on the following lemma.

Lemma 3. Consider the vector space $\left(\{0,1\}^{\lambda}, \oplus\right), \lambda \geq 2$, with basis $\mathcal{B}=\left\{a_{1}, \ldots\right.$, $\left.a_{\lfloor\lambda / 2\rfloor}, b_{1}, \ldots, b_{\lceil\lambda / 2\rceil}\right\}$. Let $A$ be equal to the linear subspace generated by the vectors in $\mathcal{B}_{A}=\left\{a_{1}, \ldots, a_{\lfloor\lambda / 2 \mid}\right\}$, and let $B$ be the linear subspace generated by the vectors in $\mathcal{B}_{B}=\left\{b_{1}, \ldots, b_{\lceil\lambda / 2\rceil}\right\}$. Define $S:=A \cup B$. Then it holds that:

(i) Any vector $z \in\{0,1\}^{\lambda}$ can be expressed as $z=a \oplus b$ with $a, b \in S$, and this expression (i.e., the vectors $a$ and $b$ ) can be found efficiently (i.e., in at most poly $(\lambda)$ steps).

(ii) For all distinct vectors $x_{0}, x_{1}, v \in\{0,1\}^{\lambda}$ there is an equal number of combinations of linear subspaces $A$ and $B$ as defined above for which $x_{0} \oplus v \in A$ and $x_{1} \oplus v \in B$.

(iii) $S$ has cardinality $|S| \leq 2 \cdot 2^{\lceil\lambda / 2\rceil}$.

Proof. (i) Notice that any vector $z \in\{0,1\}^{\lambda}$ can be expressed as a linear combination of all basis vectors: $z=\sum u_{i} a_{i}+\sum v_{j} b_{j}$, i.e., $z=a \oplus b$ with $a \in A$ and $b \in B$. This expression is found efficiently by using Gaussian elimination.

(ii) Without loss of generality, since $x_{0}, x_{1}$ and $v$ are distinct vectors, we may choose $a_{1}=x_{0} \oplus v \neq 0$ and $b_{1}=x_{1} \oplus v \neq 0$. The number of combinations of linear subspaces $A$ and $B$ is independent of the choice of $a_{1}$ and $b_{1}$. (Notice that if $x_{0} \neq x_{1}$ but $v=x_{0}$, then the number of combinations is twice as large.)

(iii) The bound follows from the construction of $S$ and the cardinalities of $A$ and $B$, which are $|A|=2^{\lfloor\lambda / 2\rfloor}$ and $|B|=2^{\lceil\lambda / 2\rceil}$.

An Example. Let us give an example in order to illustrate the principle of Lemma 3. Consider the vector space $\left(\{0,1\}^{\lambda}, \oplus\right)$ for an even $\lambda$, and choose as subbases $\mathcal{B}_{A_{0}}=$ $\left\{e_{1}, \ldots, e_{\lambda / 2}\right\}$ and $\mathcal{B}_{B_{0}}=\left\{e_{\lambda / 2+1}, \ldots, e_{\lambda}\right\}$, where $e_{i}$ is the unit vector of length $\lambda$ that has a one in position $i$ and zeros in all other positions. Then the basis $\mathcal{B}_{A_{0}}$ spans the subspace $A_{0}$ that contains all vectors of length $\lambda$ whose second half is all zero, and $\mathcal{B}_{B_{0}}$ spans the subspace $B_{0}$ that comprises all vectors of length $\lambda$ whose first half is all zero. It then follows immediately that every vector $z \in\{0,1\}^{\lambda}$ can be expressed as $z=a \oplus b$ with $a \in A_{0}$ and $b \in B_{0}$, or, saying this differently, with $a, b \in S$ and $S:=A_{0} \cup B_{0}$. It is also immediate that $S$ has cardinality $|S| \leq 2 \cdot 2^{\lambda / 2}$.

Relevance for PUFs. The lemma translates into a PUF context as follows. Suppose that a malicious and an honest player play the following game. The malicious player gets access to a PUF with challenge length $\lambda$ in an initialization period, in which he can query CRPs of his choice from the PUF. After that, the PUF is taken away from 
him. Then, the honest player chooses a vector $z \in\{0,1\}^{\lambda}$ and sends it to the malicious player. The malicious player wins the game if he can present the correct PUF-responses $r_{0}$ and $r_{1}$ to two arbitrary challenges $c_{0}$ and $c_{1}$ which have the property that $c_{0} \oplus c_{1}=z$. Our lemma shows that in order to win the game with certainty, the malicious player does not need to read out the entire CRP space of the PUF in the initialization phase; he merely needs to know the responses to all challenges in the set $S$ of Lemma 3, which has a quadratically reduced size compared to the entire CRP space. This observation is at the heart of the attack described below.

In order to make the attack hard to detect for the honest player, it is necessary that the attacker chooses random subspaces $A$ and $B$, and does not use the above trivial choices $A_{0}$ and $B_{0}$ all the time. This fact motivates the random choice of $A$ and $B$ in Lemma 3 . The further details are as follows.

The Attack. As in [12], we assume that the PUF has got a challenge set of $\{0,1\}^{\lambda}$. Given Lemma 3 the OT-receiver (who initially holds the PUF) can achieve a quadratic advantage in Protocol 1 as described below.

First, he chooses uniformly random linear subspaces $A$ and $B$, and constructs the set $S$, as described in Lemma 3. While he holds possession of the PUF before the start of the protocol, he reads out the responses to all challenges in $S$. Since $|S| \leq 2 \cdot 2^{\lceil\lambda / 2\rceil}$, this is a quadratic improvement over reading out all responses of the PUF.

Next, he starts the protocol as normal. When he receives the two values $x_{0}$ and $x_{1}$ in Step 3 of the protocol, he computes two challenges $c_{0}^{*}$ and $c_{1}^{*}$ both in set $S$ such that

$$
x_{0} \oplus x_{1}=c_{0}^{*} \oplus c_{1}^{*} .
$$

According to Lemma 3 i), this can be done efficiently (i.e., in poly $(\lambda)$ operations). Notice that, since the receiver knows all the responses corresponding to challenges in $S$, he in particular knows the two responses $r_{0}^{*}$ and $r_{1}^{*}$ that correspond to the challenges $c_{0}^{*}$ and $c_{1}^{*}$.

Next, the receiver deviates from the protocol and sends the value $v:=c_{0}^{*} \oplus x_{0}$ in Step 4. For this choice of $v$, the two challenges $c_{0}$ and $c_{1}$ that the sender uses in Step 5 satisfy

$$
c_{0}:=c_{0}^{*} \oplus x_{0} \oplus x_{0}=c_{0}^{*}
$$

and

$$
c_{1}:=c_{0}^{*} \oplus x_{0} \oplus x_{1}=c_{0}^{*} \oplus c_{0}^{*} \oplus c_{1}^{*}=c_{1}^{*} .
$$

By Lemma 3 (ii), Alice cannot distinguish the received value $v$ in Step 4 from any random vector $v$. In other words, Alice cannot distinguish Bob's malicious behavior (i.e., fabricating a special $v$ with suitable properties) from honest behavior. As a consequence, Alice continues with Step 6 and transmits $S_{0}=s_{0} \oplus r_{0}^{*}$ and $S_{1}=s_{1} \oplus r_{1}^{*}$. Since Bob knows both $r_{0}^{*}$ and $r_{1}^{*}$, he can recover both $s_{0}$ and $s_{1}$. This breaks the security of the protocol.

Please note the presented attack is simple and effective: It fully works within the original communication model of Brzuska et al. [12]. Furthermore, it does not require laborious computations of many days on the side of the attacker (as certain modeling attacks on PUFs do [25]). Finally, due to the special construction we proposed, the 
honest players will not notice the special choice of the value $v$, as the latter shows no difference from a randomly chosen value.

Effect on Bit Commitment (Protocol2). Due to the reductionist construction of Protocol 2, our attack on the oblivious transfer scheme of Protocol 1 directly carries over to the bit commitment scheme of Protocol 2 if Protocol 1 is used in it as a subprotocol. By using the attack, a malicious sender can open the commitment in both ways by reading out only $2 \cdot 2^{\lceil\lambda / 2\rceil}$ responses (instead of all $2^{\lambda}$ responses) of the PUF. On the other hand it can be observed easily that the hiding property of the BC-Protocol2 2 is unconditional, and is not affected by our attack.

\section{Practical Consequences of the Attack}

What are the practical consequences of our quadratic attack, and how relevant is it in real-world applications? The situation can perhaps be illustrated via a comparison to classical cryptography. What effect would a quadratic attack have on schemes like RSA, DES and SHA-1? To start with RSA, the effect of a quadratic attack here is rather mild: The length of the modulus must be doubled. This will lead to longer computation times, but restore security without further ado. In the case of single-round DES, however, a quadratic attack would destroy its security, and the same holds for SHA-1. The actual effect of our attack on PUF-based OT and BC has some similarities with DES or SHA-1: PUFs are finite objects, which cannot be scaled in size indefinitely due to area requirements, arising costs, and stability problems. This will also become apparent in our subsequent discussion.

\subsection{Electrical Integrated PUFs}

We start our discussion by electrical integrated PUFs, and take the well-known Arbiter PUF as an example. It has been discussed in theory and realized in silicon mainly for challenge lengths of 64 bits up to this date [9]10 15]28]. Our attack on such a 64-bit implementation requires the read-out of $2 \cdot 2^{32}=8.58 \cdot 10^{9} \mathrm{CRPs}$ by the receiver. This read-out can be executed before the protocol (i.e., not during the protocol), and will hence not be noticed by the sender. Assuming a MHz CRP read-out rate [15] of the Arbiter PUF, the read-out takes $8.58 \cdot 10^{3} \mathrm{sec}$, or less than $144 \mathrm{~min}$.

Please note that the attack is independent of the cryptographic hardness of the PUF, such as its resilience against machine learning attacks. For example, a 64-bit, 8-XORArbiter PUF (i.e., an Arbiter PUF with eight parallel standard 64-bit Arbiter PUFs whose single responses are XORed at the end of the structure) is considered secure in practice against all currently known machine learning techniques [25]. Nevertheless, this type of PUF would still allow the above attack in $144 \mathrm{~min}$.

Our attacks therefore enforce the use of PUFs with a challenge bitlength of 128 bits or more in Protocols 1 and 2, Since much research currently focuses on 64-bit implementations of electrical PUFs, publication and dissemination of the attack seems important to avoid their use in Protocols 1 and 2 Another aspect of our attack is that it motivates the search for OT- and BC-protocols that are immune, and which can safely 
be used with 64-bit implementations. The reason is that the usage of 128-bit PUFs doubles the area consumption of the PUF and negatively affects costs.

\subsection{Optical PUFs}

Let us now discuss the practical effect of our attack on the optical PUF introduced by Pappu [20] and Pappu et al. [21]. The authors use a cuboid-shaped plastic token of size $1 \mathrm{~cm} \times 1 \mathrm{~cm} \times 2.5 \mathrm{~mm}$, in which thousands of light scattering small spheres are distributed randomly. They analyze the number of applicable, decorrelated challengeresponse pairs in their set-up, arriving at a figure of $2.37 \cdot 10^{10}$ [21]. Brzuska et al. assume that these challenges are encoded in a set of the form $\{0,1\}^{\lambda}$, in which case $\lambda=\left\lceil\log _{2} 2.37 \cdot 10^{10}\right\rceil=35$. If this number of $2^{35}$ is reduced quadratically by virtue of Lemma 3, we obtain on the order of $2 \cdot 2^{18}=5.2 \cdot 10^{5}$ CRPs that must be read out by an adversary in order to cheat. It is clear that even dedicated measurement setups for optical PUFs cannot realize the $\mathrm{MHz}$ rates of the electrical example in the last section. But even assuming mild read-out rates of 10 CRPs or 100 CRPs per second, we still arrive at small read-out times of $5.2 \cdot 10^{4} \mathrm{sec}$ or $5.2 \cdot 10^{3} \mathrm{sec}$, respectively. This is between 14.4 hours (for 10 CRPs per second) or 87 minutes (for 100 CRPs per second). If a malicious receiver holds the PUF for such a time frame before the protocol starts (which is impossible to control or prevent for the honest players), he can break the protocol's security.

Can the situation be cleared by simply scaling the optical PUF to larger sizes? Unfortunately, also an asymptotic analysis of the situation shows the same picture. All variable parameters of the optical PUF [21|20|16] are the $x-y$-coordinate of the incident laser beam and the spatial angle $\Theta$ under which the laser hits the token. This leads to a merely cubic complexity in the three-dimensional diameter $d$ of the cuboid scattering token. 2 Given our attack, this implies that the adversary must only read out $O\left(d^{1.5}\right)$ challenges in order to cheat in Protocols 1 and 2. If only the independent challenges are considered, the picture is yet more drastic: As shown in [31], the PUF has at most a quadratic number of independent challenges in $d$. This reduces to a merely linear number of CRPs which the adversary must read out in our attack. Finally, we remark that scaling up the size of the PUF also quickly reaches its limits under practical aspects: The token considered by Pappu et al. [2120] has an area of $1 \mathrm{~cm} \times 1 \mathrm{~cm}$. In order to slow down the quadratic attack merely by a factor of 10 , a token of area $10 \mathrm{~cm} \times 10 \mathrm{~cm}$ would have to be used. Such a token is too large to even fit onto a smart card.

Overall, this leads to the conclusion that optical PUFs like the ones discussed in [20]21 16] cannot be used safely with the Protocols 1 and2] in the face of our attack. Due to their low-degree polynomial CRP complexity, and due to practical size constraints, simple scaling of the PUFs constitutes no efficient countermeasure. This distinguishes the optical approach from the electrical case of the last section. This observation has a particular relevance, since Brzuska et al. had explicitly suggested optical PUFs for the implementation of their protocols (see Section 8 of [2]).

\footnotetext{
${ }^{2}$ Please note in this context that the claim of [2] that the number of CRPs of an optical PUF is super-polynomial must have been made erroneously or by mistake; our above brief analysis shows that it is at mostly cubic. The low-degree polynomial amount of challenges of the optical PUF is indeed confirmed by the entire literature on the topic, most prominently [21,20]31].
} 


\section{Potential Countermeasures}

\subsection{Additional PUF Transfers and Time Constraints?}

Can we bind the time in which the malicious player has got access to the PUF in order to prevent our attack? The current Protocols 1 and 2 obviously are unsuited to this end; but could there be modifications of theirs which have this property? A simple approach seems the introduction of one additional PUF transfer from the sender to the receiver in the initialization phase. This assumes that the sender initially holds the PUF, transfers it to the receiver, and measures the time period within which the receiver returns the PUF. The (bounded) period in which the receiver had access to the PUF can then be used to derive a bound on the number of CRPs the receiver might know. This could be used to enforce security against a cheating receiver. Please note that a long, uncontrolled access time for the sender is no problem for the protocol's security, whence it suffices to concentrate on the receiver.

On closer inspection, however, there are significant problems with this approach. In general, each PUF-transfer in a protocol is very costly. One PUF-transfer per protocol seems acceptable, since it is often executed automatically and for free, for example by consumers carrying their bank cards to cash machines. But having two such transfers in one protocol, as suggested above, will most often ruin a protocol's practicality.

A second issue is that binding the adversarial access time in a tight manner by two consecutive PUF transfers is very difficult. How long will one physical transfer of the PUF take? 1 day? If the adversary can execute this transfer a few hours faster and can use the gained time for executing measurements on the PUF, our countermeasure fails. The same holds if the adversary carries out the physical transfer himself and can measures the PUF while it is in transit.

In summary, enforcing a tight time bound on the receiver's access time by two PUF transfers or also by other measures will be impossible in almost any applications. The above idea may thus be interesting as a theoretical concept for future PUF-protocol design, but cannot be considered a generally efficient and practically relevant countermeasure.

\subsection{Interactive Hashing}

Let us now discuss a second and more effective countermeasure: The employment of interactive hashing $(\mathrm{IH})$ as a substep in OT protocols. As we will show, protocols based on IH can achieve better security properties than Protocol 1 The idea of using IH in the context of PUFs has been first been suggested by Rührmair in 2010; his OT-protocol was the first published PUF-based two-player protocol [23]. The following approach is a simplified version of his original scheme. We also give (for the first time) a security analysis of the protocol. Via the general reduction of BC to OT presented in Protocol2, our construction for OT can also be used to implement PUF-based BC.

\subsubsection{Interactive Hashing as a Security Primitive}

Interactive hashing $(\mathrm{IH})$ is a two-player security primitive suggested by [18]17]. It has been deployed as a protocol tool in various contexts, including zero-knowledge proofs, 
bit commitment and oblivious transfer (see references in [17]. The following easily accessible and application-independent definition of IH has been given in [4]; for more a formal treatment see [27].

Definition 4 (Interactive Hashing (IH) [4]). Interactive Hashing is a cryptographic primitive between two players, the sender and the receiver. It takes as input a string $c \in\{0,1\}^{t}$ from the sender, and produces as output two t-bit strings, one of which is $c$ and the other $c^{\prime} \neq c$. The output strings are available to both the sender and the receiver, and satisfy the following properties:

1. The receiver cannot tell which of the two output strings was the original input. Let the two output strings be $c_{0}, c_{1}$, labeled according to lexicographic order. Then if both strings were a priori equally likely to have been the sender's input $c$, then they are a posteriori equally likely as well.

2. When both participants are honest, the input is equally likely to be paired with any of the other strings. Let $c$ be the sender's input and let $c^{\prime}$ be the second output of interactive hashing. Then provided that both participants follow the protocol, $c^{\prime}$ will be uniformly distributed among all $2^{t}-1$ strings different from $c$.

3. The sender cannot force both outputs to have a rare property. Let $\mathcal{G}$ be a subset of $\{0,1\}^{t}$ representing the sender's "good set". Let $G$ be the cardinality of $\mathcal{G}$ and let $T=2^{t}$. Then if $G / T$ is small, the probability that a dishonest sender will succeed in having both outputs $c_{0}, c_{1}$ be in $\mathcal{G}$ is comparably "small".

One standard method to implement IH is by virtue of a classical technique by Naor et al. [17]. To achieve a self-contained treatment, we describe this technique in a variant introduced by Crepeau et al. [4] below. In the protocol below, let $c$ be a $t$-bit string that is the input to sender in the interactive hashing. All operations take place in the binary field $\mathcal{F}_{2}$.

\section{Protocol 5: Interactive Hashing [4]}

1. The receiver chooses a $(t-1) \times t$ matrix $\mathbf{Q}$ uniformly at random among all binary matrices of rank $t-1$. Let $q_{i}$ be the $i$-th query, consisting of the $i$-th row of $\mathbf{Q}$.

2. For $1 \leq i \leq t-1$ do:

(a) The receiver sends query $q_{i}$ to the sender.

(b) The sender responds with $v_{i}=q_{i} \cdot c$.

(c) Given $\mathbf{Q}$ and $v \in\{0,1\}^{t-1}$ (the vector of the sender's responses), both parties compute the two values of $c \in\{0,1\}^{t}$ consistent with the linear system $\mathbf{Q} \cdot c=$ $v$. These solutions are labeled $c_{0}, c_{1}$ according to lexicographic order.

The following theorem, which is taken from [4|27], tells us about the security of the above scheme. It relates to the security definition 4

Theorem 6 (Security of Protocol 5). Protocol 5 satisfies all three information theoretic security properties of Definition 4 Specifically, for Property 3 of Definition 4 it ensures that a dishonest sender can succeed in causing both outputs to be in the "good set" $\mathcal{G}$ with probability at most $15.6805 \cdot G / T$, where $G=|\mathcal{G}|$ and $T=2^{t}$. 


\subsubsection{Oblivious Transfer}

We are now presenting a PUF-based oblivious transfer protocol that uses IH as a substep. It bears some similarities with an earlier protocol of Rührmair [23] in the sense that it also uses interactive hashing, but is slightly simpler.

\section{Protocol 7: PUF-BASED 1-OUT-OF-2 OBLIVIOUS TRANSFER WITH INTERACTIVE HASHING}

1. The sender's input are two strings $s_{0}, s_{1} \in\{0,1\}^{\lambda}$ and the receiver's input is a bit $b \in\{0,1\}$.

2. The receiver chooses a challenge $c \in\{0,1\}^{\lambda}$ uniformly at random. He applies $c$ to the PUF, which responds $r$. He transfers the PUF to the sender.

3. The sender and receiver execute an IH protocol, where the receiver has input $c$. Both get outputs $c_{0}, c_{1}$. Let $i$ be the value where $c_{i}=c$.

4. The receiver sends $b^{\prime}:=b \oplus i$ to the sender.

5. The sender applies the challenges $c_{0}$ and $c_{1}$ to the PUF. Denote the corresponding responses as $r_{0}$ and $r_{1}$.

6. The sender sends $S_{0}:=s_{0} \oplus r_{b^{\prime}}$ and $S_{1}:=s_{1} \oplus r_{1-b^{\prime}}$ to receiver.

7. The receiver recovers the string $s_{b}$ that depends on his choice bit $b$ as $S_{b} \oplus r=$ $s_{b} \oplus r_{b \oplus b^{\prime}} \oplus r=s_{b} \oplus r_{i} \oplus r=s_{b}$.

\subsubsection{Security and Practicality Analysis}

We start by a security analysis of Protocol 7 in the so-called "stand alone, good PUF model", which was introduced by van Dijk and Rührmair in [6]. In this communication model, the following two assumptions are made: (i) the PUF-protocol is executed only once, and the adversary or malicious players have no access to the PUF anymore after the end of the protocol; (ii) the two players do not manipulate the used PUFs on a hardware level. We stress that whenever these two features cannot be guaranteed in practical applications, a number of unexpected attacks apply, which spoil the security of the respective protocols. Even certain impossibility results can be shown under these circumstances; see [6] for details.

In the following analysis in the stand alone, good PUF model, we assume that the adversary has the following capabilities:

1. He knows a certain number of CRPs of the PUF, and has possibly used them to build an (incomplete) predictive model of the PUF. In order to model this ability, we assume that there is a proper subset $S \subsetneq C$ of the set of all challenges $C$ such that the adversary knows the correct responses to the challenges in $S$ with probability one. The cardinality of $S$ depends on the previous access times of the adversary to the PUF and the number of CRPs he has collected from other sources, for example protocol eavesdropping. It must be estimated by the honest protocol users based on the given application scenario. Usually $|S| \ll|C|$.

2. Furthermore, we assume that the adversary can correctly guess the response to a uniformly and randomly chosen challenge $c \in C \backslash S$ with probability at most $\epsilon$, where the probability is taken over the choice of $c$ and over the adversary's 
random coins. Usually $\epsilon$ will be significantly smaller than one. To name two examples: In the case of a well-designed electrical PUF with single-bit output, $\epsilon$ will be around 0.5 ; in the case of a well-designed optical PUF [20[21] with multi-bit images as outputs, $\epsilon$ can be extremely small, for example smaller than $2^{-100}$. Again, the honest protocol users must estimate $\epsilon$ based on the circumstances and the employed PUF.

Assuming the above capabilities and using Theorem 6 , the probability that the receiver can cheat in Protocol/7 is bounded above by

$$
15.6805 \cdot|S| /|C|+\epsilon,
$$

a term that will usually be significantly smaller than one.

Under the presumption that this cheating probability of the receiver is indeed smaller than one, the security of Protocol 7 can be further amplified by using a well-known result by Damgard, Kilian and Savail (see Lemma 3 of [5]):

Theorem 8 (OT-Amplification [5]). Let $(p, q)$-WOT be a 1-2-OT protocol where the sender with probability $p$ learns the choice bit $c$ and the receiver with probability $q$ learns the other bit $b_{1-c}$. Assume that $p+q<1$. Then the probabilities $p$ and $q$ can be reduced by running $k(p, q)$-WOT-protocols to obtain a $\left(1-(1-p)^{k}, q^{k}\right)$-WOT protocol.

In the case of our OT-Protocol7 7 it holds that $p=0$, whence the technique of Damgard et al. leads to an efficient security amplification, and to a $\left(0, q^{k}\right)$-WOT protocol. The PUF does not need to be transferred $k$ times, but one PUF-transfer suffices. We remark that the probability amplification according to Theorem 8 is not possible with Protocol 1 after our quadratic attack, since the attack leads to a cheating probability of one for the receiver, i.e., $p+q \geq 1$ in the language of Theorem 8 .

Let us quantatively illustrate the security gain of Protocol 7 over Protocol 1 via a simplified back-of-the-envelope calculation: We argued earlier that via our quadratic attack, a malicious receiver who has read out $2 \cdot 2^{18}$ CRPs from an optical PUF can cheat with probability 1 (= with certainty) in Protocol[1 Let us compare this to the case that an optical PUF is used in the IH-based Protocol7 7 . Let us assume that the adversary has collected the same number of CRPs $\left(=2 \cdot 2^{18} \mathrm{CRPs}\right)$ as in the quadratic attack, and that the (multi-bit) response of the optical PUF on the remaining CRPs is still hard to preduct, i.e., it cannot be predicted better than with probability $\epsilon \leq 2^{-100}$. Then by Theorem 6 and by our above analysis, the adversary's chances to break Protocol 7 are merely around $15.6805 \cdot 2^{19} \cdot 2^{-35}+2^{-100} \approx 0.00024$. This probability can then be exponentially reduced further via Theorem 8 .

On the downside, however, the IH-Protocol 5 has a round complexity that is linear (i.e., equal to $\lambda-1)$ in the security parameter $\lambda$. This is relatively significant for the optical PUF (where $\lambda=35$ ) and electrical PUFs with medium bitlengths (where $\lambda=$ $64)$. One possible way to get around this problem is to use the constant round interactive hashing scheme by Ding et al. [7]. However, this scheme has slightly worse security guarantees than the IH scheme of the last sections. Future work will analyze the exact security loss under the use of the IH scheme of Ding. A first analysis to this end can be found in van Dijk and Rührmair [6]. 
To summarize the discussion in this section, interactive hashing can restore the security of PUF-based OT protocols even for small sized PUFs with 64-bit challenge lengths and for optical PUFs in the stand alone, good PUF model. Via the general reduction of $\mathrm{BC}$ to OT given in Protocol2, this result can be used to securely implement PUF-based $\mathrm{BC}$ in this model, too. However, the use of IH leads to an increased number of communication rounds that is about equal to the (binary) challenge length of the PUF, i.e., around 64 rounds for the integrated PUFs with 64 bit challenges, and around 35 rounds for optical PUFs of size $1 \mathrm{~cm}^{2}$ [21]. It must be decided on the basis of the concrete application scenario whether such a number of rounds is acceptable.

\section{Summary and Conclusions}

We revisited PUF-based OT- and BC-protocols, including the recent schemes of Rührmair from Trust 2010 [23] and Brzuska et al. from Crypto 2011 [12]. We placed special emphasis on the security which these protocols achieve in practice, in particular when they are used in connection with widespread optical and 64-bit electrical PUFimplementations. Our analysis revealed several interesting facts.

First of all, we described a simple and efficient method by which the OT- and BCprotocol of Brzuska et al. can be attacked with probability one in practice if electrical PUFs with 64-bit challenge lengths are used, or whenever optical PUFs are employed. Since much research focuses on 64-bit implementations of electrical PUFs [9 10 15], and since Brzuska et al. had explicitly suggested optical PUFs for the implementation of their protocols (see Section 8 of [2]), the publication and dissemination of our quadratic attack seems important to avoid their use in Protocols 1 and 2 . Please note that our attack is independent of the cryptographic hardness of the PUF, and is merely based on its challenge size.

Secondly, we discussed an alternative class of protocols for oblivious transfer that are based on interactive hashing techniques. They are inspired by the earlier OT-protocol of Rührmair [23]. We argued that these protocols lead to better security in practice. They can be used safely with 64-bit electrical PUFs. When used with optical PUFs, they lead to better security than the protocols of Brzuska et al., but the security margins are tighter than in the 64-bit case. In both cases, a well-known result by Damgard, Kilian and Savail [5] can be used in order to reduce the cheating probabilities exponentially.

Our discussion shows once more that PUFs are quite special cryptographic and security tools. Due to their finite nature, asymptotic constants that might usually be hidden in $O(\cdot)$ - and $\Theta(\cdot)$-notations become relevant in practice and should be discussed explicitly. Furthermore, their specific nature often allows new and unexpected forms of attacks. One of the aims of our work is to bridge the gap between PUFs in theory and applications; reconciling these two fields seems a necessary prerequisite for a healthy long-term development of the field. We hope that the general methods and the approach of this paper can contribute to this goal.

Recommendations for Protocols Use and Future Work. Let us conclude the paper with a condensed recommendation for the practical implementation of PUF-based OT and $\mathrm{BC}$ protocols, and by a discussion of future work. Firstly, it is clear from our results that 
the protocol of Brzuska et al. cannot be used safely with optical PUFs a la Pappu (i.e., with non-integrated optical PUFs that have only a small or medium sized challenge set), or with electrical PUFs with challenge lengths around 64 bits.

Secondly, we showed that Protocols based on interactive hashing (IH) can achieve better security. These protocols can be employed safely with optical PUFs and with electrical PUFs of challenge length 64. Furthermore, Damgard et al.'s [5] amplification technique can be applied in order to bring the cheating probabilities arbitrarily close to zero. Nevertheless, we would like to stress once more to practical PUF-users that this analysis only applies if the protocols are employed in the stand alone, good PUF model (see Section 5.2.3 and [6]). As soon as the features of this model cannot be enforced in a given application (for example by certifying a PUF, or by erasing PUF responses at the protocol end [6]), certain new attacks apply, which spoil both the security of IH-based protocols and of the protocols of Brzuska et al. These attacks are not the topic of this publication, but have been described in all detail in [6].

If a PUF has challenge length of 128 bits or more, it seems at first sight that the protocols of Brzuska et al. could be used safely in the stand alone, good PUF model, too, but we stress that this recommendation is yet to be confirmed by full formal analysis. One issue is that the PUF security feature required by the protocols of Brzuska et al. is (in a nutshell) that the adversary must be unable to select two PUF-challenges with a given distance $d$ such that he knows the two corresponding responses. This security property of a PUF is new in the literature and should yet be further investigated in future work before final recommendations are being made. In particular, it does not seem simple or straightforward to judge in practice whether a given PUF fulfills this property.

A second topic for future research is how the round complexity of the IH-based protocols can be reduced. Some steps to this end have been made by van Dijk and Rührmair in [6], where the constant-round interactive hashing scheme of Ding et al. [7] is applied to obtain contant-round PUF-based OT and BC protocols.

Acknowledgements. The authors would like to thank Stefan Wolf and Jürg Wullschleger for enjoyable discussions, and Stefan Wolf for suggesting the example in Section 3 page 256 to us. Part of this work was conducted within the physical cryptography project at the TU München.

\section{References}

1. Brzuska, C., Fischlin, M., Schröder, H., Katzenbeisser, S.: Physically Uncloneable Functions in the Universal Composition Framework. In: Rogaway, P. (ed.) CRYPTO 2011. LNCS, vol. 6841, pp. 51-70. Springer, Heidelberg (2011)

2. Brzuska, C., Fischlin, M., Schröder, H., Katzenbeisser, S.: Physical Unclonable Functions in the Universal Composition Framework. Full version of the paper. Available from Cryptology ePrint Archive (2011) (downloaded on February 28, 2012)

3. Canetti, R.: Universally Composable Security: A New Paradigm for Cryptographic Protocols. In: FOCS 2001, pp. 136-145 (2001); Full and updated version available from Cryptology ePrint Archive

4. Crépeau, C., Kilian, J., Savvides, G.: Interactive Hashing: An Information Theoretic Tool (Invited Talk). In: Safavi-Naini, R. (ed.) ICITS 2008. LNCS, vol. 5155, pp. 14-28. Springer, Heidelberg (2008) 
5. Damgård, I., Kilian, J., Salvail, L.: On the (Im)possibility of Basing Oblivious Transfer and Bit Commitment on Weakened Security Assumptions. In: Stern, J. (ed.) EUROCRYPT 1999. LNCS, vol. 1592, pp. 56-73. Springer, Heidelberg (1999)

6. van Dijk, M., Rührmair, U.: Physical Unclonable Functions in Cryptographic Protocols: Security Proofs and Impossibility Results. Cryptology ePrint Archive, Report 228/2012 (2012)

7. Ding, Y.Z., Harnik, D., Rosen, A., Shaltiel, R.: Constant-round oblivious transfer in the bounded storage model. Journal of Cryptology 20(2), 165-202 (2007)

8. Gassend, B.: Physical Random Functions. MSc Thesis. MIT (2003)

9. Gassend, B., Clarke, D.E., van Dijk, M., Devadas, S.: Silicon physical random functions. In: ACM Conference on Computer and Communications Security 2002, pp. 148-160 (2002)

10. Gassend, B., Lim, D., Clarke, D., van Dijk, M., Devadas, S.: Identification and authentication of integrated circuits. Concurrency and Computation: Practice \& Experience 16(11), 10771098 (2004)

11. Guajardo, J., Kumar, S.S., Schrijen, G.-J., Tuyls, P.: FPGA Intrinsic PUFs and Their Use for IP Protection. In: Paillier, P., Verbauwhede, I. (eds.) CHES 2007. LNCS, vol. 4727, pp. 63-80. Springer, Heidelberg (2007)

12. Impagliazzo, R., Rudich, S.: Limits on the Provable Consequences of One-Way Permutations. In: STOC 1989, pp. 44-61 (1989)

13. Kilian, J.: Founding cryptography on oblivious transfer. In: STOC (1988)

14. Kumar, S.S., Guajardo, J., Maes, R., Schrijen, G.J., Tuyls, P.: The Butterfly PUF: Protecting IP on every FPGA. In: HOST 2008, pp. 67-70 (2008)

15. Lee, J.-W., Lim, D., Gassend, B., Suh, G.E., van Dijk, M., Devadas, S.: A technique to build a secret key in integrated circuits with identification and authentication applications. In: Proceedings of the IEEE VLSI Circuits Symposium (June 2004)

16. Maes, R., Verbauwhede, I.: Physically Unclonable Functions: a Study on the State of the Art and Future Research Directions. In: Naccache, D., Sadeghi, A.-R. (eds.) Towards HardwareIntrinsic Security, sec. 1. Springer (2010)

17. Naor, M., Ostrovsky, R., Venkatesan, R., Yung, M.: Perfect zero- knowledge arguments for NP using any one-way permutation. Journal of Cryptology (1998); Preliminary version In: Brickell, E.F. (ed.) CRYPTO 1992. LNCS, vol. 740, pp. 196-214. Springer, Heidelberg (1993)

18. Ostrovsky, R., Venkatesan, R., Yung, M.: Fair games against an all-powerful adversary. In: AMS DIMACS Series in Discrete Mathematics and Theoretical Computer Science, pp. 155169 (1993); Preliminary version in SEQUENCES 1991

19. Majzoobi, M., Koushanfar, F., Potkonjak, M.: Lightweight Secure PUFs. In: IC-CAD 2008, pp. 607-673 (2008)

20. Pappu, R.: Physical One-Way Functions. PhD Thesis, Massachusetts Institute of Technology (2001)

21. Pappu, R., Recht, B., Taylor, J., Gershenfeld, N.: Physical One-Way Functions. Science 297, 2026-2030 (2002)

22. Rivest, R.: Illegitimi non carborundum. Invited keynote talk, CRYPTO 2011 (2011)

23. Rührmair, U.: Oblivious Transfer Based on Physical Unclonable Functions. In: Acquisti, A., Smith, S.W., Sadeghi, A.-R. (eds.) TRUST 2010. LNCS, vol. 6101, pp. 430-440. Springer, Heidelberg (2010)

24. Rührmair, U., Busch, H., Katzenbeisser, S.: Strong PUFs: Models, Constructions and Security Proofs. In: Sadeghi, A.-R., Tuyls, P. (eds.) Towards Hardware Intrinsic Security: Foundation and Practice. Springer (2010)

25. Rührmair, U., Sehnke, F., Sölter, J., Dror, G., Devadas, S., Schmidhuber, J.: Modeling Attacks on Physical Unclonable Functions. In: ACM Conference on Computer and Communications Security (2010) 
26. Rührmair, U., Sölter, J., Sehnke, F.: On the Foundations of Physical Unclonable Functions. Cryptology e-Print Archive (June 2009)

27. Savvides, G.: Interactive Hashing and reductions between Oblivious Transfer variants. $\mathrm{PhD}$ thesis, McGill University, Montreal (2007)

28. Suh, G.E., Devadas, S.: Physical Unclonable Functions for Device Authentication and Secret Key Generation. In: DAC 2007, pp. 9-14 (2007)

29. Tuyls, P., Schrijen, G.-J., Škorić, B., van Geloven, J., Verhaegh, N., Wolters, R.: Read-Proof Hardware from Protective Coatings. In: Goubin, L., Matsui, M. (eds.) CHES 2006. LNCS, vol. 4249, pp. 369-383. Springer, Heidelberg (2006)

30. Tuyls, P., Škorić, B.: Strong Authentication with Physical Unclonable Functions. In: Petkovic, M., Jonker, W. (eds.) Security, Privacy and Trust in Modern Data Management. Springer (2007)

31. Tuyls, P., Škorić, B., Stallinga, S., Akkermans, A.H.M., Ophey, W.: Information-Theoretic Security Analysis of Physical Uncloneable Functions. In: Patrick, A.S., Yung, M. (eds.) FC 2005. LNCS, vol. 3570, pp. 141-155. Springer, Heidelberg (2005) 